\title{
SHAPE MEMORY ALLOY ACTUATOR DESIGN: CASMART COLLABORATIVE BEST PRACTICES
}

\section{Othmane Benafan University of Central Florida}

\section{Travis Turner NASA Langley}

\author{
Jeff Brown \\ Dynalloy, Inc.
}

F. Tad Calkins
The Boeing Company

Parikshith Kumar

Texas A\&M University

\section{John Webster \\ Rolls-Royce PLC}

\section{ABSTRACT}

Upon examination of shape memory alloy (SMA) actuation designs, there are many considerations and methodologies that are common to them all. A goal of CASMART's design working group is to compile the collective experiences of CASMART's member organizations into a single medium that engineers can then use to make the best decisions regarding SMA system design. In this paper, a review of recent work toward this goal is presented, spanning a wide range of design aspects including evaluation, properties, testing, modeling, alloy selection, fabrication, actuator processing, design optimization, controls, and system integration. We have documented each aspect, based on our collective experiences, so that the design engineer may access the tools and information needed to successfully design and develop SMA systems. Through comparison of several case studies, it is shown that there is not an obvious single, linear route a designer can adopt to navigate the path of concept to product. SMA engineering aspects will have different priorities and emphasis for different applications.

\section{INTRODUCTION}

Shape memory alloys (SMAs) have been a topic of significant research interest for over forty years. Microstructural complexities and their resulting thermomechanical properties have presented challenges in understanding how to integrate these materials into structural applications. Consequently, design of structural and mechanical systems involving SMA materials has been sparse and ad hoc. However, upon examination of a variety of system designs, there are many considerations and parameters that are common to them all. The Consortium for the Advancement of Shape Memory Alloy Research and Technology (CASMART) was established to foster sharing of technical knowledge about SMAs, to achieve new understanding of the materials, and to facilitate progress in applying that knowledge. The Design Working Group of CASMART strives to serve as a single location to capture the collective best practices, critical information and perspective necessary for designing systems involving SMA materials and actuators.

The objective of this paper is to compile the experiences of the authors into a resource that can aid other engineers in SMA actuator development. Design aspects of particular interest include evaluation, properties, testing, modeling, alloy selection, fabrication, actuator processing, design optimization, controls, and system integration. As one might expect, these topics may have different priorities and emphasis for different applications and even for different approaches to a common application. Also, many of these topics are interrelated in the design process for typical applications.

Many aspects of SMA actuator design are described and discussed in the following sections. The discussion captures a broad perspective on development and implementation attributes that affect SMA actuator design. Interrelations between aspects are also discussed. In conclusion, several case studies are presented, highlighting the variety in design priorities and processes that arise in developing applications.

\section{EVALUATION}

The main purpose of evaluation is to answer the question: "Does SMA make sense for this application?" Distinct advantages are often found for SMAs in applications where there are space and/or weight constraints, large deformation and/or force requirements, or there is a thermal environment from which energy can be extracted for benefit. A combined use of empirical and numerical tools is the most efficient way for designers of all skill levels to assess the viability of SMA actuation for their application. These tools are documented in subsequent sections.

Evaluating the suitability of SMA can vary from application to application. The following 8 questions provide signposts that can direct the evaluation process.

1. Why use shape memory alloys? The shape memory behavior of interest must be identified, i.e., the shape memory effect or superelasticity. This behavior should have potential advantages relative to other solutions and technologies.

2. What are the application requirements? These requirements will identify what properties and performance are needed from the SMA element. Applications can require the SMA element to provide a single operational cycle up to millions of cycles. Life of an SMA component is driven by the desired response time and thermo-mechanical loading conditions. These requirements in turn drive the choice of material, form, size, and control methods. 
3. What are the cost/expenditure limits? The costs include the raw SMA material, processing, and fabrication. The resulting cost per device is critical to the business case for many applications to be commercially viable.

4. What is the availability and size of the SMA element? The available input (power) required output (work) of the SMA element often drives material and form selection. SMA elements can be of a variety of forms (strips, rods, sheets, wires, springs, tubes) in various sizes. Another key consideration is the availability and required volume of the material from a commercial supplier or other source.

5. What efficiency and response time is needed? Both energy and mechanical efficiencies of SMA components may be drivers in evaluating SMA technology. However, many applications, particularly in the aerospace industry, are weight sensitive; thus weight savings (mass efficiency) may be of higher priority. The maximum available cyclic frequency can also drive the evaluation.

6. What is the proposed environment? The environment, in particular thermal conditions, will greatly influence evaluation of SMAs, especially if commercially availability of alloys is a factor. Currently, transformation temperatures are limited to $\sim 100{ }^{\circ} \mathrm{C}$, but this is expected to increase in the near future. Other concerns may include vibration, humidity, corrosive elements, and bio-compatibility.

7. What relevant standards and documents are available? This is especially critical in applications that require certification. Examples of such documents include ASTM standards: F2004-05, F2005-05, F2063-05, F2082-06, F2516-07, F2633-07, application specific documents, certification documentation, and supplier data.

8. What other components/system will be required? Finally, the SMA element is often one component in a system that can include other mechanical and electrical elements. One must consider if required system integration technologies (See System Considerations) are available or if development is needed, potentially resulting in increased application cost.

Once SMAs are deemed an advantageous technology for the application, a more detailed design process begins with some combination of the aspects described in the next sections. Often, a "zero-order" model can be assembled from answering these evaluation questions and carried into this development.

\section{ALLOY SELECTION}

The first consideration in development of an SMA actuator is typically material selection. Material selection should be based upon application constraints and requirements including displacement, force, ambient and actuator temperatures, corrosion, cyclic stability and fatigue life. Factors that affect alloy selection are discussed in this section.

SMA materials are generally produced by conventional vacuum melting technologies such as VAR (vacuum arc remelting), VIM (vacuum induction melting), and ISM (induction skull melting). There is significant variety in the composition and properties of known and available SMA materials. Compositional variation results in fundamentally different thermo-mechanical behaviors, and these typically drive alloy selection. The baseline SMA is Nitinol, which is an alloy consisting of near-equal proportions of Nickel and Titanium. The most common and easily procured alloys are based on the 55-Nitinol (equiatomic, 55-wt\% NiTi) composition. Another well-established, but less common group of alloys is based on Ni-rich 60-Nitinol composition. More recently, Ni-rich 57-Nitinol alloys have become available and have grown in interest. In addition to binary alloys, NiTiFe, an alloy which enhances R-phase (see "Case Studies: Thermal Switching"), is readily available on a production level. Although not currently available due to demand, NiTiCu, an alloy which exhibits low thermal hysteresis and improved functional fatigue, has been produced on a production level in the past. In the near future, higher transformation temperature materials, such as Ni-Ti-Pd, Ni-Ti-Pt and Ni-Ti-Hf are expected to be available. These ternary alloys will open doors for hightemperature SMA applications, such as aircraft engine components. Other less-commonly used materials include $\mathrm{Cu}$ and Fe-based alloys.

Matching the transformation temperatures of an SMA to the application is another crucial step in alloy selection. Some general rules of thumb with regard to transformation temperatures follow. It is important to realize in the following discussion that all transformation temperatures increase with applied stress (see "Testing and Properties"). The austenite finish temperature should be less than the application temperature range $\left(A_{f}<T\right)$ in cases where superelasticity is of interest. Such cases include those that require energy dissipation and/or large deformation at relatively constant, and perhaps low, loads. The application temperature range should bound the transformation temperatures $\left(T_{l}<M_{f}, A_{f}<T_{h}\right.$ Figure 1) for maximizing actuator work output in passivecontrol cases that employ environmental heating and cooling. For cases where environmental temperatures do not vary significantly or the variation is unsuitable for passive control, the application temperature should be less than the martensite finish temperature $\left(T<M_{f}\right)$ to maximize work output via control by applied heating and environmental cooling.

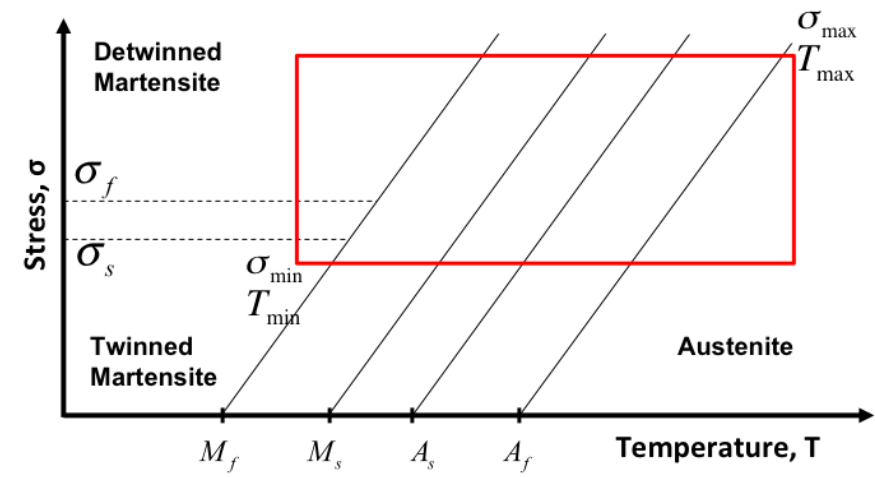

Figure 1: Stress-temperature phase diagram - red box bounds a typical actuator design space. Consideration of transformation temperatures relative to environmental temperatures is crucial to successful alloy selection. 
Transformation strain is another principal factor in the selection of an SMA composition. Near-equiatomic, 55-Nitinol exhibits the greatest recoverable strain capability of commercially produced SMA materials for both stress-induced transformation (superelasticity) and the thermoelastic shape memory effect. Strains approaching $10 \%$ can be achieved for single or very low-cycle applications. Strains on the order of 4$5 \%$ can be achieved for applications requiring durability and reliability for tens-of-thousands of cycles, with further strain limitations leading to even better functional fatigue behavior. Ni-rich alloy chemistries, such as 57- and 60-Nitinol, have improved thermomechanical stability relative to nearequiatomic materials, but at the expense of less strain capability in the range of $1-1.5 \%$. Similarly, the ternary alloys (NiTiHf, NiTiPd and NiTiPt) offer increased application temperatures, but compromise on strain range (3-4\% max).

The amount and character of the hysteresis inherent to an SMA varies with alloy chemistry and processing and, thus, it can be an important factor in alloy selection. SMAs exhibiting high hysteresis are particularly useful for energy dissipation applications. Applications where cyclic response involving large stroke or strain is anticipated, such as the seismic response of buildings, are suited to hysteretic energy dissipation via SMAs. Hysteresis can pose significant challenges in other applications, such as those involving active control.

It is often desirable to heat SMA actuators or components by directly applied electrical current. In such cases, NiTi alloys are advantageous relative to $\mathrm{Cu}$-based SMAs because of their relatively high resistivity, being comparable to Nichrome. High resistivity may also be helpful for in-situ resistance measurement for feedback control. Low resistivity may be advantageous when uniform actuator temperature or conduction heat loss to the surroundings is important.

Other factors affecting alloy selection are often equally important to those already discussed, including operating stress, yield stress, desired form, commercial availability, required processing, shape setting methods, cold work, heat treatment, machining, wire drawing, melting, joining, cost, life, development cost/time, previous experience, available data, composition repeatability and sensitivity to microstructural variability. Material properties are available in the literature and also from suppliers for many SMA materials. Use of those properties is implied in the previous discussion. Many properties of SMA materials are sensitive to processing, applied thermo-mechanical parameters, and loading history. Thus, preliminary testing of the selected material may be necessary to ensure proper alloy selection for application-specific actuator development. Discussion of test methods and material/actuator properties is found in "Testing and Properties."

\section{ACTUATOR DEVELOPMENT}

Once the material has been selected and fundamental properties of the material are deemed appropriate for the application, development of the actuator can commence. This process typically involves selection of an actuator form, processing the actuator to refine thermo-mechanical performance, and testing to quantify the performance over the parametric space anticipated in the application.

\section{Fabrication}

Fabrication of SMA actuators requires consideration of 1) actuator form and functionality, 2) fabrication methods, 3) joining techniques, 4) system integration and 5) interrelation between these four aspects, as shown in Figure 2. An advantage of SMAs is that the same material can usually be processed into various forms, the most common of which are wire, ribbon, ring, bar, rod, foil, sheet, spring, tube, and plate. Fabrication of SMA mill products are produced by conventional metalworking processes such as forging, rolling, and drawing. Depending on the form, SMA actuators can generate motion in one dimension, such as in the case of a thin-wire form, in two dimensions, such as in bending of a bar or even motion in a more complex threedimensional manner, such as in honeycomb or micro-bump structures. Form selection is dependent on element functionality or actuation method. Tension (e.g., wires, springs), compression (e.g., rods, springs), bending (e.g., beams, plates) and torsion (e.g., rods, tubes, and springs) loading modes have all been exploited in some form based upon the desired functionality.

\begin{tabular}{|c|c|}
\hline \multicolumn{2}{|c|}{ Fabrication } \\
\hline Actua & Fabrication methods \\
\hline & \multirow{4}{*}{$\begin{array}{l}\text { Material removal: } \\
\text { Mechanical machining (milling, } \\
\text { drilling, turning) } \\
\text { Laser beam /water jet } \\
\text { Etching /electropolishing } \\
\text { Electrical discharge machining } \\
\text { (EDM) } \\
\text { Deformation processes: } \\
\text { Hot forming (forging, rolling, } \\
\text { extrusion) } \\
\text { Cold forming (wire drawing, } \\
\text { swaging) } \\
\text { Consolidation processes } \\
\text { Powder metallurgy/sputtering } \\
\text { Bonding }\end{array}$} \\
\hline $\begin{array}{l}\text { Forms: } \\
\text { Wire/bar/sheet/spring/tube } \\
\text { /plate/beam/ribbons/3D } \\
\text { Function: } \\
\text { Linear (tension/compression) } \\
\text { /bending/torsion }\end{array}$ & \\
\hline Joining techniques & \\
\hline $\begin{array}{l}\text { Welding } \\
\text { Arc/plasma/laser/resistance } \\
\text { Soldering/brazing } \\
\text { Mechanical joining } \\
\text { Fasteners/press fit/shrink fit } \\
\text { Crimping }\end{array}$ & \\
\hline $\begin{array}{l}\text { Adhesive } \\
\text { Bonding }\end{array}$ & System integration \\
\hline & $\begin{array}{l}\text { Attachment location } \\
\text { Finishing } \\
\text { Assembly }\end{array}$ \\
\hline
\end{tabular}

Figure 2: SMA actuator fabrication processes.

Screening methods can be used to rule out fabrication processes using a fabrication-processes-first approach [1, 2]. This approach helps in eliminating the fabrication processes that will not satisfy the actuator development requirement. Such requirements could be geometric complexities, actuator size or production volume. Figure 2 identifies three major fabrication method categories. Material removal processes including mechanical machining (milling, drilling and turning), laser or water-jet cutting, etching/electropolishing and electrical discharge machining (EDM) are processes that generally 
involve wasted material that increases the total fabrication cost. Deformation processes including hot forming (e.g., forging, rolling, extrusion) and cold forming (e.g., wire drawing, swaging) are processes that change the shape of the raw material by rearranging the solid. Consolidation processes such as powder metallurgy, sputtering, plasma spray and bonding are processes that combine powder or small sub-parts together.

Actuators with complex shape and properties often involve more than one fabrication process. Sometimes, complete fabrication requires joined assemblies of two or more components. Various joining techniques are available but only a few have been shown to be effective, as shown in Figure 2. Welding SMA to SMA can be effective, but also problematic. Besides being a monolithic process, common concerns associated with welding are brittleness, thermal-induced residual stresses and changes in properties. Nitinol can be welded to itself using traditional spot welding techniques. Acceptable joint strengths can be achieved when the welding is done in an inert-gas environment, e.g., argon, to avoid formation of brittle oxides. Soldering and brazing are other commonly adopted joining techniques. Brazing has been demonstrated as an effective method to join Nitinol to itself through the use of a Ag-rich barrier layer to prevent the formation of brittle intermetallics like $\mathrm{NiTi}_{2}$ [3]. Soldering/brazing fluxes reduce concerns about the heat affected zone and provide closer assembly tolerances and a neater joint appearance. However, the composition, strength and melting point of the assembly can be altered. When permanent joints are to be avoided, mechanical fastening is an option. Several mechanical fasteners are available which include integral fasteners (e.g., flanges, embossed protrusions and crimps), discrete fasteners (e.g., screws, inserts, studs, rivets), seams, press fits, shrink/expansion fits and more. Although this joining method provides easy disassemble and reassemble without affecting the base material properties, concerns such as misalignment, stress concentrations and environmental conditions can be an issue.

\section{Actuator Processing}

In conjunction with the fabrication and joining processes presented above, actuator structure and properties can be further tuned through processing. Actuator processing serves to change component structure or properties, or to restore those that were altered during fabrication. Common metallurgical techniques may be employed to achieve some of these adjustments, such as strain hardening, solid-solution strengthening, precipitation strengthening, grain size refinement and dispersion hardening. In nearly all actuator applications, however, heat treatments and thermo-mechanical training are employed, thus they are the focus of this section.

A variety of heat treatments may be used to alter actuator structure and properties. Shape setting is a thermo-mechanical process that nearly every actuator goes through. A common practice for shape setting entails 1) mechanically constraining the fabricated material (wire, sheet, tube, etc.) within a fixture, die or mandrel of the desired shape, 2) heat treating the constrained material at a high temperature for a dwell time (commonly at $500{ }^{\circ} \mathrm{C}$ for $1 \mathrm{hr}$ for the NiTi), 4) cooling the material (vacuum, air, or quenching) and finally 5) optimizing the final geometry through subsequent shape sets or additional machining. In addition to shape setting, heat treatments are often used to elicit precipitate structures that effectively tune properties such as yield strength, dimensional stability, and transformation temperatures, or to relieve internal stresses.

In addition to heat treatments, thermo-mechanical training processes are used to establish stabilized actuator performance for application-specific operational and environmental conditions. These training processes often entail a series of load-bias cycles (see Testing and Properties) designed to impart cycle-to-cycle mechanical stability, a property that dramatically influences functional fatigue of the actuator. This is critical to fail-safe design, safe operation, reliable performance and the life of the application.

\section{TESTING AND PROPERTIES}

Following determination of actuator configuration, operating temperatures (see Material Selection), and operating loads of the actuator, the design space for the application can be represented on a stress-temperature phase diagram (Figure 1). Examining the actuation behavior of the SMA component within this space can facilitate component design and integration. To do this, though, critical material properties are required. These properties can be broadly categorized as:

1. Effective thermo-elastic properties;

2. Critical stresses and temperatures for transformation;

3. Other properties and additional factors.

In the remainder of this section, definitions and methods for obtaining these properties are reviewed.

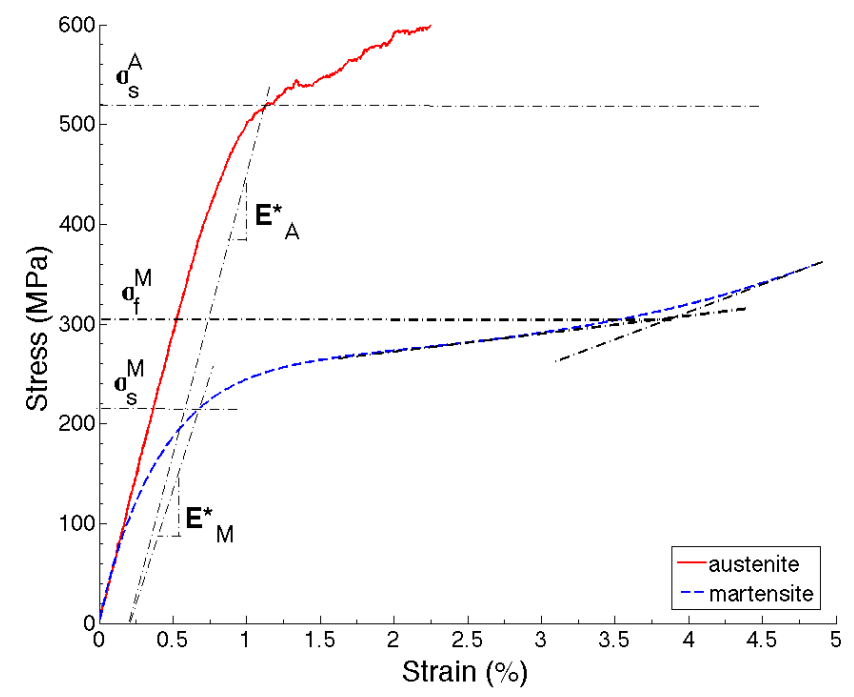

Figure 3: Example of effective elastic property evaluation.

\section{Effective Thermo-elastic Properties}

While inelastic mechanisms contributing to SMA properties differ [4-6], their effective properties are analogous to those of other structural materials. Thus, graphical methods similar to those used for other materials may be incorporated. Assuming isotropic material behavior, the effective Young's 
modulus $\left(E^{*}\right)$ of the austenitic and martensite phases can be determined from fitting the initial loading responses of each phase, as shown in Figure 3. The effective thermal expansion coefficients of the material, $\alpha_{\mathrm{A}}^{*}$ and $\alpha_{\mathrm{M}}{ }_{\mathrm{M}}$, can be measured from observing the pure austenite and pure martensite straintemperature slopes of a zero load load-bias test. "Load-bias" testing refers to a test in which a specimen is thermally cycled through phase transformation under constant applied stress [7]. Representative responses are shown in Figure 4. Traditionally, effective Poisson's ratios $\left(v_{\mathrm{A}}{ }_{\mathrm{A}}, v_{\mathrm{M}}{ }_{\mathrm{M}}\right)$ have been assumed $[8,9]$, though recent measurements of Poisson's ratios have been reported for NiTi $[6,10,11]$.

\section{Critical Transformation Stresses and Temperatures}

Knowledge of transformation temperatures and critical stresses, as well as temperature-stress relationships is essential for actuator development. The analog of yield stress determination for non-SMA materials may be incorporated to determine some critical stresses. That is, a $0.2 \%$ strain offset method can be used to determine the onset stress for reorientation of the martensite phase $\left(\sigma^{\mathrm{M}}{ }_{\mathrm{s}}\right)$ and the onset stress for stress-induced transformation of the austenite phase $\left(\sigma^{\mathrm{A}}{ }_{\mathrm{s}}\right)$, as shown in Figure 3. To determine other inflection points, it is often common practice to adopt the "tangent intersection method" [12]. For example, in Figure 3 this method is used to observe the stress at which martensite reorientation begins to saturate $\left(\sigma_{\mathrm{f}}^{\mathrm{M}}\right)$. These initial and saturation stresses are $\sigma_{\mathrm{s}}$ and $\sigma_{\mathrm{f}}$ on the phase diagram (Figure 1). Generally speaking, they are significant to actuation application design since below $\sigma_{\mathrm{s}}$, very little transformation strain will be exhibited by the SMA component, and above $\sigma_{\mathrm{f}}$, maximum transformation strain will be achieved. There are exceptions and limitations to these generalities - for instance, through the two-way shape memory effect transformation strain can be achieved at low stresses. Also, SMA performance will start to deteriorate if the material is subjected to stresses moderately higher than $\sigma_{\mathrm{f}}$.

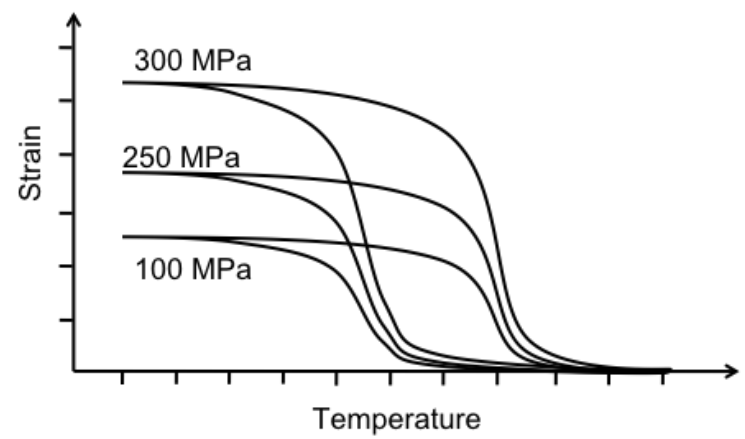

Figure 4: Load-bias tests at different stress levels.

In Figure 1, the four surfaces that represent the phase transformation regions separate the pure austenite phase from the martensite phase. These transformation surfaces can be linear, quadratic or of a higher order non-linear form depending on the material system, processing and thermo-mechanical treatment. For simplicity, they are often approximated as linear, as in Figure 1, and each surface is characterized by the slope relating the shift of a transformation temperature with different levels of applied stress. These slopes are described by "Clausius-Clapeyron" coefficients (e.g., $\mathrm{C}_{\mathrm{A}}, \mathrm{C}_{\mathrm{M}}$ ). Stress-free transformation temperatures $\left(\mathrm{M}_{\mathrm{s}}, \mathrm{M}_{\mathrm{f}}, \mathrm{A}_{\mathrm{s}}, \mathrm{A}_{\mathrm{f}}\right)$ are traditionally determined by conducting a simple Differential Scanning Calorimetry (DSC) analysis [13]. However, for developing an actuator application, it may be desirable to instead determine them through a very small load-bias test $\left(\sigma_{\text {eng }}<10 \mathrm{MPa}\right)$. In choosing the latter method, the same specimen may then be used for a series of load-bias tests, as shown in Figure 4. From this series, the tangent intersection method may be used to determine transformation temperatures and their relationship to applied stress (hence $\mathrm{C}_{\mathrm{A}}, \mathrm{C}_{\mathrm{M}}$ ).

\section{Additional Properties and Other Factors}

Additional tests to determine performance of the material/system under shock loads or in a corrosive environment may be required, depending on the requirements of the application. In all SMA testing and data interpretation, it is important to understand the processing history (see Actuator Processing) of the component and consider effects it may have on the observed responses. Heat treatments, machining processes, specimen geometry, and cold work all have the ability to alter SMA behaviors, thus care must be taken in interpreting data for application design. While fundamental tests and material property determination can aid application design, it is often advantageous to use some combination of modeling and empirical observation of the desired SMA component performance for design validation.

\section{PARAMETRIC INVESTIGATION}

Proper use of numerical tools can reduce the development times and costs of shape memory alloy components. In a product development cycle, there are several occasions where it may be advantageous to perform a calculation or simulation. The earliest is in the "evaluation" stage. Models can provide a means for investigating whether or not an SMA is able to deliver a desired performance. However, these tools are perhaps even greater assets in the material, processing, configuration, and topological parameter selection processes.

Readily available tools for calculating SMA behaviors include manufacturer supplied material properties (e.g., [14]), engineering models (e.g., [8, 15, 16], and SMA constitutive models (e.g., [9, 17-25]). These tools vary in complexity, capability, and accuracy. Knowing how to select the most efficient tool for a desired outcome requires some knowledge of both shape memory alloy behaviors and the tools themselves. As documentation of these tools grows, the tool selection process becomes easier. Parametric design tools may be coupled with the SMA models to optimize actuator parameters at a level that, in most cases, is not practical to achieve solely through empirical studies.

\section{Models}

First Order Calculations

If commercially supplied material is being used, first-order parametric evaluation may be performed through calculations using vendor-supplied properties and equations. In some 
instances, such as in wire design, this may be sufficient for the entire project. For example, if buying actuator wire from Dynalloy, their "Tech Sheets" document [14] provides both electrical and mechanical properties for their entire family of alloys used in six common wire actuator configurations. These sheets provide all of the information necessary to select the proper wire diameter, length, and biasing component.

\section{Engineering Models}

Where first order calculations do not produce the desired level of certainty, accuracy, or resolution, engineering models are often developed from empirical data to facilitate selection of final design parameters. One common way to develop an engineering model is to first create SMA test specimens in the likeness of a non-SMA mechanical component that is thought to be of use for the application (such as a spooled wire [15] or a helical spring [16]). Then the response of each the austenite and martensite phases of the SMA in this configuration are observed through testing. To create the numerical model, parameters are fit and/or new parameters are introduced to the engineering or graphical model for the analogous non-shape memory component such that the empirical response of each phase is replicated, and the difference between the two provides the transformation properties.

\section{Constitutive Models}

Many different constitutive models have been developed to simulate SMA behaviors over the course of the past 30 years. These range from microstructure models that can be used to study and predict effects of composition and processing (e.g., [23]) to phenomenological continuum models with a wide variety of features developed specifically for engineering components and devices (e.g., [9, 17-20, 22]). Models of the latter class are traditionally implemented into commercial finite element packages to facilitate component simulations. With proper parameterization, they can predict performance of nearly any configuration without the need for empirical observation.

The CASMART Modeling Working Group is documenting methodologies for comparing SMA constitutive models to facilitate model selection [26]. In short, the designer should first understand SMA characteristics that are critical to component performance. Then, the simplest model capable of replicating these characteristics should be selected. For instance, if designing a rod actuator only subjected to tension, a one-dimensional model should be chosen as opposed to a threedimensional model. Similarly, if designing a porous structure, a three-dimensional model formulated to handle phenomena associated with highly localized stress concentrations would be desired. Benefits beyond one-time simulation can be obtained by linking the finite element simulations with design software packages, as discussed in the next section.

\section{Design Optimization}

By applying methods of design optimization, the analysis tools can guide engineers and designers to the best configurations and parameters. In component design, the full potential of SMA models is realized when the models are linked with some type of optimization algorithm to enable parametric study on a scale and at speeds that are not practical solely through experimentation. For first-order calculations and engineering models, this may be done through a spreadsheet or numerical algorithm. In the design of structural components using constitutive models, a complementary design software package may be selected and integrated with the model to automate simulations and explore a design space subjected to specified optimization parameters.

\section{SYSTEM CONSIDERATIONS}

Final design of an SMA actuator requires consideration of system integration factors. Such factors may include the manner in which the actuator is joined with the rest of the system, thermal management, and control methodology.

\section{Fabrication and Integration}

Integration of SMA actuators into structural and/or mechanical systems can involve many factors. Perhaps foremost in importance is consideration of whether the SMA actuator is distinct from (attached only discretely) or integrated with (attached, bonded or embedded in a continuous manner) other parts of the system. This SMA actuator integration attribute affects many other system integration factors.

Attachment provisions are a primary concern in either the distinct or integrated actuator case. Options include mechanical connections, bonding, welding and brazing. Mechanical connections are the simplest type and are most common in applications involving distinct SMA actuators or where electrical connection is desired. Mechanical connections and techniques for joining SMA to SMA were discussed in the "Actuator Development-Fabrication" section. Similar techniques involving mechanical connections, welds or brazed joints are often used when integrating the actuator into a mechanical or structural system. An exception is when the actuator must be bonded to or embedded within a fiberreinforced polymeric (FRP) structure.

Techniques used for bonding an actuator to the exterior of a structure and embedding one within a polymer-based composite structure are similar. Structural adhesives include epoxies, acrylics, silicones and hot melts. Bonds with adhesives and composite resins can often be rendered effective through simple mechanical means such as light sandblasting or etching to roughen and/or remove the oxide layer. Even greater bond strengths can be achieved through a more sophisticated process of etching and priming the SMA actuator to achieve a combined mechanical and chemical bond. As with every other joining method, adhesive bonding disadvantages consist of poor impact properties, instability at relatively low temperatures and chemical reactions [27].

In many cases, SMA actuators work against a deformable structure or articulating mechanism. Although two-way shape memory can be implemented to enable reversible control of an actuated system, greater stroke and force can be achieved by employing two one-way shape memory elements in agonistantagonist pairs and/or by using a non-SMA bias element in the system. Implementation of agonist-antagonist pairs can be 
complicated by the need for thermal isolation between actuators. The bias-element approach is more common and can be achieved via a conventional spring or a deforming structure, both of which provide a restoring force to return the SMA actuator to the rest configuration.

Heating by direct current requires access to the actuator ends and is dependent upon electrical resistance (see Alloy Selection) that is compatible with the power source. Actuator access locations and electrical resistance can both be controlled as desired by forming electrical circuits via joints (e.g., mechanical connectors, welds, brazing) between multiple SMA actuators. Other means of active applied heating include surface-mounted and cartridge heaters that can be bonded or inserted into SMA actuators.

For direct-current heating, power transmission wires should be routed as directly as possible to minimize resistive load and wire gauge should be adequate to carry the required current to the SMA actuators without introducing parasitic losses. Connectivity to the SMA actuators can be achieved through mechanical connections or a better and more permanent electrical connection can be achieved through soldering. An acceptable solder joint requires cladding (e.g., $\mathrm{Cu}$ ) of the SMA actuator. $\mathrm{Cu}$ cladding can be accomplished by standard industrial electroplating processes. Although the impedance of SMA actuators can be controlled by development of clever circuitry, as mentioned above, the load is primarily resistive and requires a power source that can deliver adequate current for heating.

The rate at which heat can be dissipated from the SMA actuators to the environment is the dominant factor in determining frequency response of the system. Environmental heat loss can be improved by optimizing conductive, convective and, to a lesser extent, radiative heat loss paths. This must be balanced, however, with requirements for heating the actuators. Devices that are useful for improving heat loss include finned heat sinks, heat pipes and thermoelectric modules. Improvements in frequency response can be achieved by a thermal control approach involving active cooling as well as heating. Active cooling is possible through thermoelectric modules and directed forced convection.

Mechanisms have an important role in many applications. Levers and linkages are most prevalent due to the ability to amplify stroke or force. Locks, brakes and over-center devices are other mechanisms that have proven useful in conjunction with SMA actuators for some applications.

\section{Controls}

A critical element of an SMA actuation system is control methodology. Control of SMA actuators is typically implemented by active or passive means of applying and removing heat. Perhaps the most elegant applications of SMA actuators are those that use changes in ambient conditions to control the SMA actuation. More typically, however, control is achieved through applied heat and environmental cooling. It is often desirable and practical to apply heat by direct current (Joule heating) because of its simplicity and rapid response.
Active control methods include open-loop and closed-loop strategies. Closed-loop strategies require measurement of relevant parameters, which could include actuator temperature, structural or mechanical displacement, structural strain, etc. Non-contacting temperature measurement can be very valuable due to the low heat capacity of small SMA actuators. Fiber optic strain measurement is also desirable due to the low thermal sensitivity. Many other application-specific parameters may be useful for active control, such as fluid parameters (temperature, pressure) for a system involving convective heating and cooling or dynamic fluid loading.

A well-designed control system can have numerous advantages for the SMA system. It enables multiple output states such as position, mitigates some failure modes, and allows the designer to optimize the SMA performance and system design. Parts of the SMA control system include the SMA element (wire, tube, etc.), thermal elements (heating and cooling elements), sensors (output displacement sensor), and controller software and hardware. The control system design is intimately tied to the actuation form and actuation mode. The model of this SMA element is challenging because of the inherent non-linearity and hysteretic relationship between input (temperature) and output (strain). Conventional control hardware and controller designs, such as Proportional-IntegralDerivative computer based control, are often implemented for SMA applications. However, thermal management is a feature of SMA control that is not significant for many other actuators. Efficient thermal management is critical to the application success and should be one of the driving design criteria for the control system. The controller design is based on the system objectives, e.g. to minimize settling time, avoid overshoot, minimize power requirement, among others.

\section{CASE STUDIES}

The ensuing case studies demonstrate different actuator design processes performed by CASMART member organizations. In each review, the incorporation of design aspects and key findings thought to influence future SMA actuator development are summarized.

\section{Active Jet Engine Chevron}

Chevrons have been proven effective for reduction of jet exhaust noise. However, parametric effects of jet-engine chevron systems were poorly understood at the time of this work. The objective of this work was to develop active chevron technology to enable immersible chevron systems for studies of immersion amount and circumferential distribution in a wind tunnel at model scale.

Testing of active chevron systems at model scale in a wind tunnel presented some unique challenges. The model was approximately 1:9 scale of a commercial transport aircraft engine. The trailing-edge thickness was of paramount importance due to scaling issues in the aeroacoustic noise production mechanisms. Actuation via SMA materials was the only technology known to have potential for achieving a deformable structure with the thickness constraint at the evaluation stage of this application. Additionally, the 
temperature range and required cyclic frequency were within acceptable limits for known SMA materials and actuator forms.

The trailing-edge thickness requirement was achieved by embedding SMA strip (rectangular cross section wire) actuators between layers of electrically-insulating, glass-epoxy prepreg material. Actuator material with a thickness near that of the prepreg material and with a near-equiatomic NiTi composition was selected due to the thickness constraint, as well as results from preliminary testing of the material. Joule heating was planned to avoid the additional thickness of a surface-mounted heater. To allow for this type of heating, chevron-root access to the actuators was achieved by joining (welding) multiple actuator lengths to form a desirable circuit configuration.

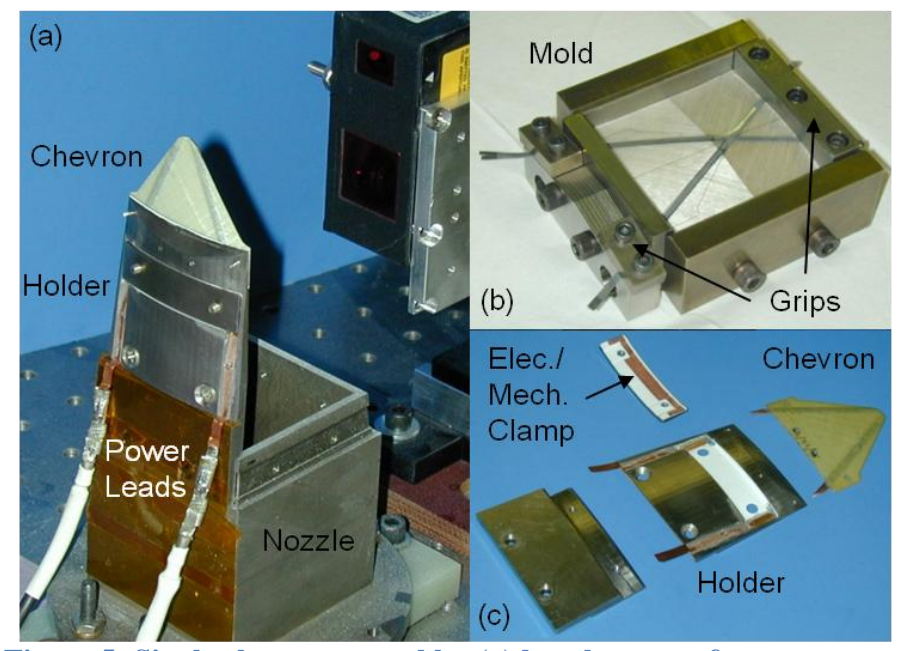

Figure 5: Single chevron assembly: (a) bench-top performance testing; (b) chevron fabrication; (c) assembly components.

Tests performed on the SMA actuator material included DSC (see "Testing and Properties") to determine the unconstrained transformation temperatures, tensile tests at a range of temperatures and blocked-force tests [16] as a function of temperature and thermal cycle. These tests supported evaluation of the concept, first-order calculations for initial design, and "calibration" of numerical models for detailed design. The evolutionary behavior of the actuator material was quantified and used directly in lieu of training, as the consolidation cure temperature was high enough to jeopardize thermo-mechanical stabilization measures performed a priori.

A constitutive model that is particularly suited to modeling SMA actuators embedded in a composite structure was selected for detailed design of the chevrons [17, 28]. Computational results helped guide fabrication of chevron prototypes and were correlated with experimental results to make changes to the design or fabrication approach.

Fabrication of the chevrons involved a vacuum-hot-press approach for consolidation of the hybrid composite structure. The actuators were constrained at the "root" and "tip" ends of the mold during consolidation to prevent recovery of the prestrain. The final chevron shape was machined from the consolidated laminates, thereby machining through one end of the actuators but leaving electrical connectivity through welds located near the free end of the chevron. The actuator ends protruding from the root end of the chevrons were $\mathrm{Cu}$-plated to improve electrical connectivity.

A PID-type controller was developed using structural strain, actuator temperature and flow properties (i.e., temperature and pressure), to control the immersion of the chevrons. The chevrons were individually fabricated and fastened to the nozzle with clamping-type mechanical/electrical connections to the protruding SMA actuators at each chevron root to allow for interchangeability and ease of replacement. Power and instrumentation wiring was integrated into the nozzle hardware. Additional details are available in [29].

\section{High Temperature SMA Springs}

The design process of high-temperature $\left(\sim 300^{\circ} \mathrm{C}\right.$ actuation temperature) SMA springs at NASA Glenn Research Center (GRC) was somewhat different from a typical actuator design process [30, 31] in that it did not begin with a specific application in mind, but rather was motivated by the desire to understand the characteristic responses of a newly developed alloy put into a structural configuration. Furthermore, many of the first-order SMA spring guidelines, such as suggested maximum shear stress and geometric deformation limits [8, 16], were not adopted in designing actuator test sequences. As a result, first-order helical actuator calculations were found inadequate for describing the observed responses. This emphasized the need for a three-dimensional material constitutive model capable of simulating SMA structural responses when loads drive them outside of a small deformation regime [30]. See "Parametric Investigation" for discussion of first-order calculations and models.

Also of interest was the documentation of spring performance vs. wire performance for this alloy (Table 1). In examining this table, the force-stroke tradeoffs are clear. Wires can provide an order of magnitude greater forces, but that same wire turned into a spring can lead to over $1500 \%$ increase in stroke, though the material is not used as efficiently, mechanically speaking, due to non-uniform loading within the structure (hence lower total work output).

Table 1: Stroke and work capabilities of wires and springs [30].

\begin{tabular}{|c|c|c|c|c|c|c|}
\hline \multicolumn{7}{|c|}{ Wire vs. Spring Capabilities } \\
\hline $\begin{array}{c}\text { Wire } \\
\text { Diameter } \\
(\mathrm{mm})\end{array}$ & $\begin{array}{c}\text { Wire } \\
\begin{array}{c}\text { Length } \\
(\mathrm{mm})\end{array}\end{array}$ & $\begin{array}{c}\text { Wire } \\
\text { Stroke } \\
(\mathrm{mm})\end{array}$ & $\begin{array}{c}\text { Wire } \\
\text { Work } \\
\text { Output } \\
\left(\mathrm{N}^{*} \mathrm{~mm}\right)\end{array}$ & $\begin{array}{c}\text { Spring } \\
\text { OD } \\
(\mathrm{mm})\end{array}$ & $\begin{array}{c}\text { Spring } \\
\text { Stroke } \\
(\mathrm{mm})\end{array}$ & $\begin{array}{c}\text { Spring } \\
\text { Work } \\
\text { Output } \\
\left(\mathrm{N}^{*} \mathrm{~mm}\right)\end{array}$ \\
\hline 0.5 & 400 & $0->9$ & 637 & 6.4 & $0->150$ & 220 \\
\hline 1.0 & 400 & $0->9$ & 2366 & 9.5 & $0->150$ & 1140 \\
\hline 1.5 & 400 & $0->9$ & 6553 & 12.7 & $0->150$ & 2570 \\
\hline
\end{tabular}

Although it was not their original intent, these components did benefit a specific application: a surge-control device for a T700 helicopter engine [31]. In this application, pneumatic actuators were not acceptable due to their bulk and additional weight of pneumatic lines and an air compressor. In wire form, $455 \mathrm{~mm}$ of this high temperature alloy was required to achieve the $3.2 \mathrm{~mm}$ stroke needed for the surge-control device, but the compartment to house the device could not accommodate this length of wire. This same stroke as well as the $22.25 \mathrm{~N}$ of force 
required for actuation of the surge-control mechanism could be provided by a single coil of a $1.5 \mathrm{~mm}$ wire diameter, $12.7 \mathrm{~mm}$ coil diameter spring made from this alloy. This spring only needs $7.5 \mathrm{~mm}$ of length to operate, making it an ideal candidate for the application given the spatial restrictions of this engine and low force requirement of the actuator.

\section{Oxygen Mask Deployment Latch Mechanism}

To promote future SMA technology, Dynalloy sponsors SMA student design projects. Boeing has partnered with Dynalloy to sponsor teams of Northwestern University freshmen in designing a lightweight oxygen mask deployment mechanisms using SMA technology. The projects have been successful, as students have achieved over $75 \%$ weight reduction from the existing pneumatic cylinder release mechanism. Once implemented, this leads to an approximate weight savings of 10 pounds per aircraft, or $\sim \$ 150,000$ savings per vehicle each year the aircraft is in service [32].

In these projects, evaluation and alloy selection phases had already been performed for the students a priori - namely, Boeing targeted these latches for weight reduction through application of SMAs, and Dynalloy had provided the students with Flexinol ${ }^{\circledR}$ wires. The students used first-order calculations aided by Dynalloy's Technical Data Sheets [14] to understand the performance strengths and tradeoffs of each configuration. For instance, they learned that if they fixed the ends of the wire and pulled on the wire's side they could achieve a larger stroke (14\% vs. $5 \%$ max), but the wire could only accommodate a fraction of the operating force $(\sim 20 \%)$, relative to an axially loaded wire. They also noted the electrical power requirements for using Joule heating to achieve actuation varied depending on wire length (higher resistance the longer the length) and diameter (higher resistance the smaller the diameter).

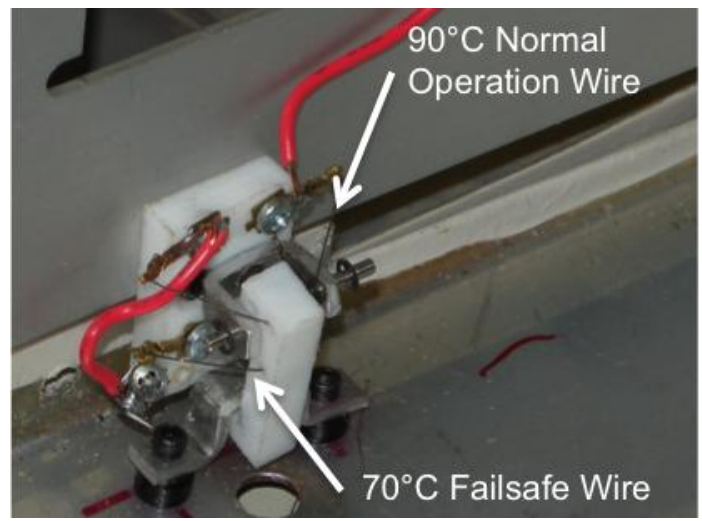

Figure 6: Picture of the $\mathrm{O}_{2}$ mask deployment mechanism.

One interesting challenge often made to SMA actuation was addressed in these design projects. Skeptics of the technology often look at the potential for unintended actuation due to abnormal heating of the wire environment. In the case of an oxygen deployment system, this was deemed a critical point, as one would not want the masks to deploy and emit oxygen in the case of a cabin fire. To address this challenge, the students made use of Dynalloy's two families of Flexinol ${ }^{\circledR}$ wire - the $70^{\circ} \mathrm{C}$ and $90^{\circ} \mathrm{C}$ wires. They used a large, $70^{\circ} \mathrm{C}$ wire as a blocking mechanism for releasing the oxygen masks, and a smaller $90^{\circ} \mathrm{C}$ as the normal-operation actuator (Figure 6). Thus, if the cabin were on fire, the larger wire would actuate first and prevent the normal operation release wire from being able to open the compartment and drop the masks.

\section{Thermal Switch}

The previously described design methodology was followed in developing a low temperature SMA thermal switch for meeting NASA's thermal management needs in advanced spaceport applications associated with future lunar and Mars missions. The objective of the switch was to provide on demand heat transfer to cool a liquid oxygen tank while the temperature of the surrounding space environment varies from $40 \mathrm{~K}$ to $400 \mathrm{~K}$ on the lunar surface. The inherent ability of SMAs to integrate sensing and actuation functions and to convert thermal to mechanical energy with large strokes and forces eliminated design complexities and the need for external power sources or storage devices.

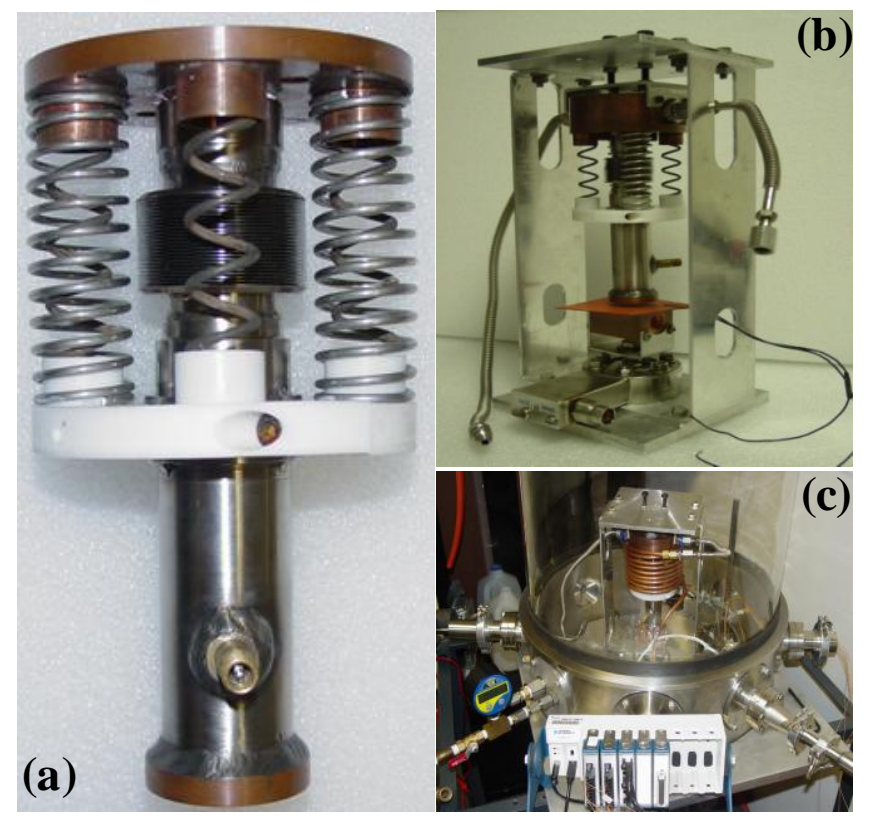

Figure 7: SMA-activated thermal switch assembly and test setup: (a) final switch assembly; (b) switch mounting fixture; (c) switch in instrumented vacuum bell jar.

A $\mathrm{Ni}_{47.1} \mathrm{Ti}_{49.6} \mathrm{Fe}_{3.3}$ alloy that exhibited a reversible phase transformation between a trigonal R-phase and a cubic austenite phase was used in order to benefit from the dimensional stability, low hysteresis and improved fatigue life typically associated with such a transformation. Since the transformation strains associated with the R-phase are low (approx. 1\%), large strokes and adequate work output were achieved by using the alloy in the form of helical springs. Heat transfer was accomplished via a wickless, closed, two-phase, variable length heat pipe with pentane and R-134a as working fluids. The heat pipe facilitated heat transfer involving two phase flow via evaporation and condensation of the working fluid. Experiments were done to quantitatively obtain 
performance parameters such as deflection curves, force output, and heat transfer rates. Testing of the switch was accomplished in a vacuum bell jar with instrumentation feedthroughs, using valves to control the flow of liquid nitrogen and heaters to simulate the temperature changes. LabVIEW® was used for data acquisition and control of the test temperature conditions. Figure 7 shows the completed thermal switch prototype. For more details, refer to [33, 34].

\section{Variable Geometry Chevron (VGC)}

The Variable Geometry Chevron (VGC) was developed to use compact, lightweight, and robust shape memory alloy actuators to morph the commercial jet engine fan chevrons between a shape optimized for noise reduction at takeoff and a shape at cruise that reduces shock cell noise without compromising engine performance [35]. In August 2005 Boeing flight-tested the VGC thrust reverser translating sleeve on an All Nippon Airway (ANA) 777-300ER with a modified commercial GE90-115B engine as part of the Quiet Technology Demonstrator 2 program. The full flight design included 14 chevrons integrated into the trailing edge of a GE90-115B thrust reverser's acoustic panel as shown in Figure 8 [35]. The VGC system was tested on six flights over five days with three different engine configurations. Autonomous operation and individual control of the 14 VGCs was demonstrated. Nine different chevron configurations were examined, investigating both community noise and shock cell noise performance. The system was able to smoothly and quickly move between immersion configurations at cruise conditions, allowing a parametric study of chevron shapes for shock cell noise reduction. Test configurations included both uniform and azimuthally varying immersion configurations. The flight test validated that the technology could be safely integrated into the airplane's structure and flight system. It represented a large step forward in the realization of SMA actuators for aerospace applications.

In examining the VGC system design, several design aspects are emphasized. The flight test actuators were a Ni-rich alloy in a bending (flexure) form. Through the material selection process, $\mathrm{Ni}$-rich $\mathrm{Ni}$-Ti alloys were chosen since they exhibit excellent thermo-mechanical stability and their transformation temperatures can be set by a heat treatment process. As a result, they do not require cold-work which allows for hot forming of complex shapes. In the actuator development phase, the flexure structure was determined to have great advantages. To evaluate the flexure configuration, Boeing has fabricated flexures of up to 10 " long, 1.5" wide with complex varying cross-sections. It was found that flexures integrate well into aerospace structures and provide a simple method of transferring large forces and moments. Regarding component integration, low profile methods of attaching a flexure to a structure may be used, including threaded holes in the SMA component to accept bolts or simple end brackets to encapsulate the flexure. These result in a low part count actuator system with reduced maintenance requirements.
Several processing methods were employed in fabricating the final flexures. The NiTi ingot was hot rolled into nominally $0.635 \mathrm{~cm}(0.25$ ") thick plate. The flexure profile was formed in a series of water jet and wire EDM machining steps. The final tapered shape minimized the size and weight of the actuators to fit into the available space while still providing the desired forces and displacements. Two holes were cut and tapped at the midpoint of each actuator for fastening to the chevron substrate. Two separate heat treatments were used to set the austenitic shape and set the actuator's transformation temperatures. Prior to installation, each actuator was conditioned for 100 thermal mechanical cycles resulting in a very stable shape change over many cycles.

The VGC program went from initial concept to a full-scale flight test in 3.5 years. The design process only made use of empirical design, fabrication, and testing iteration cycles. This was a time consuming and expensive method. Subsequently, 3D finite element modeling accurately captured the performance of the VGC [36, 37]. Currently, this model is being coupled with design optimization software to reduce development times and costs for future actuator systems [38].

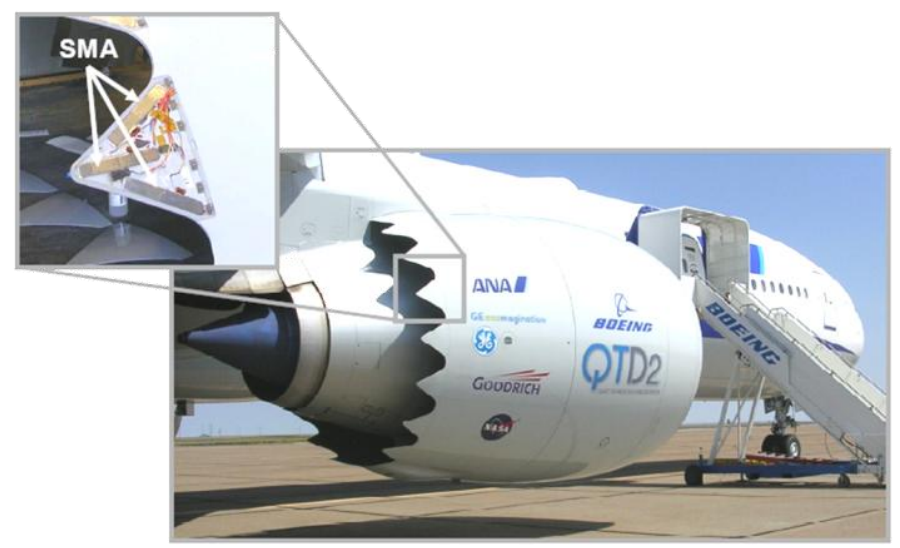

Figure 8: The GE90-115B with 14 SMA-motivated variable geometry chevrons achieved flight test August, 2005.

\section{ACKNOWLEDGMENTS}

This work documents many years of efforts, encompassing support from numerous programs and funding agencies, and we thank them all. We also acknowledge CASMART's member institutions for their support of our consortium.

\section{REFERENCES}

1. Dixon, J. and C. Poli, Engineering Design and Design for Manufacturing. 1995, Conway, MA: Field Stone Publishers.

2. Eggert, R.J., Engineering Design. 2005, Upper Saddle River NJ: Pearson Prentice Hall.

3. Shiue, R.H. and S.K. Wu, Infrared brazing of Ti50Ni50 shape memory alloy using two $\mathrm{Ag}-\mathrm{Cu}-\mathrm{Ti}$ active braze alloys. Intermetallics, 2006. 14(6): p. 630-638.

4. Rajagopalan, S., A.L. Little, M.A.M. Bourke, and R. Vaidyanathan, Elastic modulus of shape-memory NiTi from in situ neutron diffraction during macroscopic loading, instrumented indentation, and extensometryl. App. Phys. Let., 2005. 86(8).

5. Qiu, S., V.B. Krishnan, S.A. Padula, R.D. Noebe, D.W. Brown, B. Clausen, and R. Vaidyanathan, Measurement of the lattice plane 
strain and phase fraction evolution during heating and cooling in shape memory NiTi. Applied Physics Letters, 2009. 95(14).

6. Qiu, S., B. Clausen, S.A. Padula, R.D. Noebe, D.J. Gaydosh, and R. Vaidyanathan, On elastic moduli and elastic anisotropy in polycrystalline martensitic NiTi. Acta Materialia, 2011. in press.

7. Padula, S.A., D.J. Gaydosh, R.D. Noebe, G.S. Bigelow, A. Garg, D.C. Lagoudas, I. Karaman, and K.C. Atli. Influence of test procedures on the thermomechanical properties of a 55NiTi shape memory alloy. 2008. San Diego, CA: SPIE.

8. Otsuka, K. and C.M. Wayman, Shape Memory Materials. 1998, Cambridge: Cambridge University Press.

9. Lagoudas, D., Shape memory alloys : modeling and engineering applications. 1st ed. 2008, New York: Springer.

10. Stebner, A., X. Gao, D.W. Brown, and L.C. Brinson, Neutron diffraction studies and multivariant simulations of shape memory alloys: Empirical texture development - mechanical response relations of martensitic nickel-titanium. Acta Materialia, 2011. 59(7): p. 2841-2849.

11. Young, M., M.-X. Wagner, J. Frenzel, W. Schmahl, and G. Eggeler, Phase volume fractions and strain measurements in an ultrafine-grained NiTi shape-memory alloy during tensile loading. Acta Materialia, 2010. 58: p. 2344-2354.

12. ASTM, Standard Test Method for Determination of Transformation Temperature of Nickel-Titanium Shape Memory Alloys by Bend and Free Recovery. 2010, ASTM International: West Conshohocken, PA.

13. ASTM, Standard Test Method for Transformation Temperature of Nickel-Titanium Alloys by Thermal Analysis. 2010, ASTM International: West Conshohocken, PA.

14. Dynalloy, I., Technical Characteristics of Flexinol Actuator Wires. 2011: Tustin, CA.

15. Redmond, J.A., D. Brei, J. Luntz, A.L. Browne, and N.L. Johnson, Behavioral model and experimental validation for a spoolpackaged shape memory alloy actuator - art. no. 693004. Industrial and Commercial Applications of Smart Structures Technologies 2008, 2008. 6930.

16. Duerig, T.W., Engineering aspects of shape memory alloys. 1990, London ; Boston: Butterworth-Heinemann.

17. Turner, T.L., A new thermoelastic model for analysis of shape memory alloy hybrid composites. Journal of Intelligent Material Systems and Structures, 2000. 11(5): p. 382-394.

18. Hartl, D., G. Chatzigeorgiou, and D. Lagoudas, ThreeDimensional Modeling of Rate-Dependent Deformation in Shape Memory Alloys at High Temperatures. Smasis2009, Vol 1, 2009: p. 309-316.

19. Panico, M. and L.C. Brinson, A three-dimensional phenomenological model for martensite reorientation in shape memory alloys. Journal of the Mechanics and Physics of Solids, 2007. 55(11): p. 2491-2511.

20. Seelecke, S., Modeling the dynamic behavior of shape memory alloys. International Journal of Non-Linear Mechanics, 2002. 37(8): p. 1363-1374.

21. Chemisky, Y., A. Duval, B. Piotrowski, T.B. Zineb, V. Tahiri, and E. Patoor, Numerical tool for SMA material simulation: application to composite structure design. Smart Materials \& Structures, 2009. 18(10).

22. Saleeb, A.F., S.A. Padula, and A. Kumar, A multi-axial, multimechanism based constitutive modelf or the comprehensive representation of the evolutionary response of SMAs under general thermomechanical loading conditions. International Journal of Plasticity, 2011. 27: p. 655-687.
23. Gao, X. and L.C. Brinson, A Simplified Multivariant SMA Model Based on Invariant Plane Nature of Martensitic Transformation. Journal of Intelligent Material Systems and Structures, 2002. 13.

24. Lagoudas, D.C., P.B. Entchev, P. Popov, E. Patoor, L.C. Brinson, and X.J. Gao, Shape memory alloys, Part II: Modeling of polycrystals. Mechanics of Materials, 2006. 38(5-6): p. 430-462.

25. Patoor, E., D.C. Lagoudas, P.B. Entchev, L.C. Brinson, and X.J. Gao, Shape memory alloys, Part I: General properties and modeling of single crystals. Mechanics of Materials, 2006. 38(56): p. 391-429.

26. Benafan, O., L.C. Brinson, F.T. Calkins, Y. Chemisky, D.J. Hartl, D.C. Lagoudas, S. Padula, S. Seelecke, A. Stebner, and T.L. Turner. Development of Frameworks for Comparing Shape Memory Alloy Models: Macro-scale Phenomenological Continuum Models. in SMASIS. 2011. Phoenix, AZ: ASME.

27. Black, J.T., E.P. DeGarmo, and R.A. Kohser, DeGarmo's Materials and Processes in Manufacturing, ed. 10. 2007, New York, NY: John Wiley and Sons.

28. Turner, T.L., Analysis of SMAHC Structures in MSC.Nastran and ABAQUS, http://stabserv.larc.nasa.gov/smahc/, 2006.

29. Turner, T.L., R.H. Cabell, R.J. Cano, and R.J. Silcox, Development of a Preliminary Model-Scale Adapative Jet Engine Chevron. AIAA Journal, 2008. 46(10): p. 2545-2557.

30. Stebner, A., S. Padula, R. Noebe, B. Lerch, and D. Quinn, Development, Characterization, and Design Considerations of Ni19.5Ti50.5Pd25Pt5 High-temperature Shape Memory Alloy Helical Actuators. Journal of Intelligent Material Systems and Structures, 2009. 20(17): p. 2107-2126.

31. Stebner, A., S.A. Padula, R.D. Noebe, and D.D. Quinn, Characterization of Ni19.5Ti50.5Pd25Pt5 high-temperature shape memory alloy springs and their potential application in aeronautics - art. no. 69280X. Active and Passive Smart Structures and Integrated Systems 2008, 2008. 6928.

32. Maynard, M., To Save Fuel, Airlines Find No Speck Too Small, in New York Times. 2008: NY, NY.

33. Benafan, O. and R. Vaidyanathan. A Shape Memory Alloy Controlled Heat Pipe Based Thermal Switch. in International Mechanical Engineering Congress and Exposition. 2009. Lake Buena Vista, FL: ASME.

34. Benafan, O., W. Notardonato, B. Meneghelli, and R. Vaidyanathan. Design, Implementation, and Testing of a Variable Length Heat Pipe Switch with NiTiFe Helical Actuators for Thermal Management on the Lunar Surface. in International Conferense on Shape Memory and Superelastic Technologies. 2010. Pacific Grove, CA: ASM.

35. Calkins, F.T., J.H. Mabe, and G.W. Butler, Boeing's Variable Geometry Chevron: Morphing aerospace structures for jet noise reduction - art. no. 617100. Smart Structures and Materials 2006: Industrial and Commercial Applications of Smart Structures Technologies, 2006. 6171: p. O1710-O1710.

36. Hartl, D.J., D.C. Lagoudas, F.T. Calkins, and J.H. Mabe, Use of a Ni60Ti shape memory alloy for active jet engine chevron application: I. Thermomechanical characterization. Smart Materials \& Structures, 2010. 19(1).

37. Hartl, D.J., J.T. Mooney, D.C. Lagoudas, F.T. Calkins, and J.H. Mabe, Use of a Ni60Ti shape memory alloy for active jet engine chevron application: II. Experimentally validated numerical analysis. Smart Materials \& Structures, 2010. 19(1).

38. Hartl, D., D. Lagoudas, and F. Calkins, Advanced Methods for the Analysis, Design, and Optimization of SMA-Based Aerostructures. Smart Materials \& Structures, 2011. In Review. 